\title{
Statistical Machine Learning and Compressed Sensing Approaches for Analytical Electron Tomography - Application to Phase Change Materials
}

\author{
Martin Jacob $^{1 *}$, Loubna El Gueddari ${ }^{2}$, Gabriele Navarro ${ }^{1}$, Marie-Claire Cyrille ${ }^{1}$, Pascale Bayle- \\ Guillemaud $^{3}$, Philippe Ciuciu ${ }^{2}$ and Zineb Saghi ${ }^{1}$ \\ 1. Univ. Grenoble Alpes, CEA-LETI, Grenoble, France. \\ 2. CEA-NeuroSpin, Bât 145 Gif-sur Yvette, France. \\ 3. Univ. Grenoble Alpes, CEA-INAC, MEM, Grenoble, France. \\ * Corresponding author: martin.jacob@cea.fr
}

Analytical electron tomography aims at achieving 3D chemical characterization at the nanoscale using energy dispersive x-ray spectroscopy (EDX), electron energy loss spectroscopy (EELS), or both simultaneously, in a scanning transmission electron microscope (STEM). Using conventional spectral processing and reconstruction algorithms, these modes require both long acquisition times, and hence electron doses, to acquire high signal-to-noise ratio spectra, and a large number of spectrum images to faithfully reconstruct the structure in 3D. To avoid damaging the sample during the tomographic acquisition, it is necessary to explore advanced machine learning methods and reconstruction algorithms [1].

In this work, we apply vertex components analysis (VCA) and a wavelet-based compressed sensing (CS) algorithm for the 3D STEM-EELS analysis of a Ge-rich $\mathrm{Ge}_{2} \mathrm{Sb}_{2} \mathrm{Te}_{5}$ (GST) phase-change material. We were interested in seeing the phase separation that occurs between the Ge and the GST phases after an annealing at $450^{\circ}$. For this purpose, a needle-shaped sample was prepared by focused ion beam (FIB) and transferred onto an on-axis tomography holder. STEM-HAADF and STEM-EELS acquisitions were performed at $200 \mathrm{kV}$ using a probe-corrected FEI microscope equipped with a Gatan energy filter. 35 STEM-HAADF images and 18 STEM-EELS datacubes were acquired over a tilt range of $180^{\circ}$, with a pixel size of $0.53 \mathrm{~nm}$ and $2 \mathrm{~nm}$, respectively. The STEM-EELS datacubes had a frame size of $100 \times 75$ pixels, with an acquisition time of $0.1 \mathrm{sec} /$ pixel for the energy range of $250-2398 \mathrm{eV}$. The overlapping edges of Sb M4,5-edge (528 eV), O K-edge (532 eV) and Te $\mathrm{M}_{4,5}$-edge (572eV), and the presence of Ge in both phases make the use of classical elemental analysis difficult. We therefore used VCA to separate the different chemical components [2]. This ML method expresses the whole tomographic dataset as a linear combination of various pure components present in the dataset. As shown in Figure 1, this allowed us to separate the Ge-rich and the GST phases in the hyperspectral projections, in an unsupervised manner. The obtained tilt series were aligned and reconstructed using the classical simultaneous iterative reconstruction technique (SIRT) but also total variation minimization (TVM) which is nowadays widely used on under-sampled tomographic datasets [3]. TVM is based on the hypothesis that the reconstructed object is piece-wise constant. It also prohibits the reconstruction of samples with diffuse interfaces or smoothly varying intensity and can cause "cartoon" artifacts in the reconstruction. We therefore explored another sparsity term: the wavelet transform. The parameters were first optimized using the HAADF-STEM stack with 35 projections first, then 17 as for the STEMEELS dataset (figure 2). This method was found to be more adapted than TVM, as it allowed us to reconstruct the Ge and GST phases with both sharper edges and finer details (figure 3).

These data processing methods have been implemented in Python with the help of various open-source toolboxes, especially Hyperspy [4] and Tomopy [5]. ML and CS are both necessary for AET to be more widely used, but they need important parameters tuning, which determination is crucial to obtain reliable 
results. Strengths and weaknesses of these techniques will be assessed using well-known structures, and extended to EDX and simultaneous EELS-EDX datasets.

References:

[1] SM Collins and PA Midgley, Ultramicroscopy 180 (2017), p. 133.

[2] N Dobigeon and N Brun, Ultramicroscopy 120 (2012), p. 25.

[3] R Leary et al., Ultramicroscopy 131 (2013), p. 70.

[4] Hyperspy v1.4.1, http://hyperspy.org.

[5] D Gürsoy, et al., J. Synchrotron Radiat. 21 (2014), p 1188.
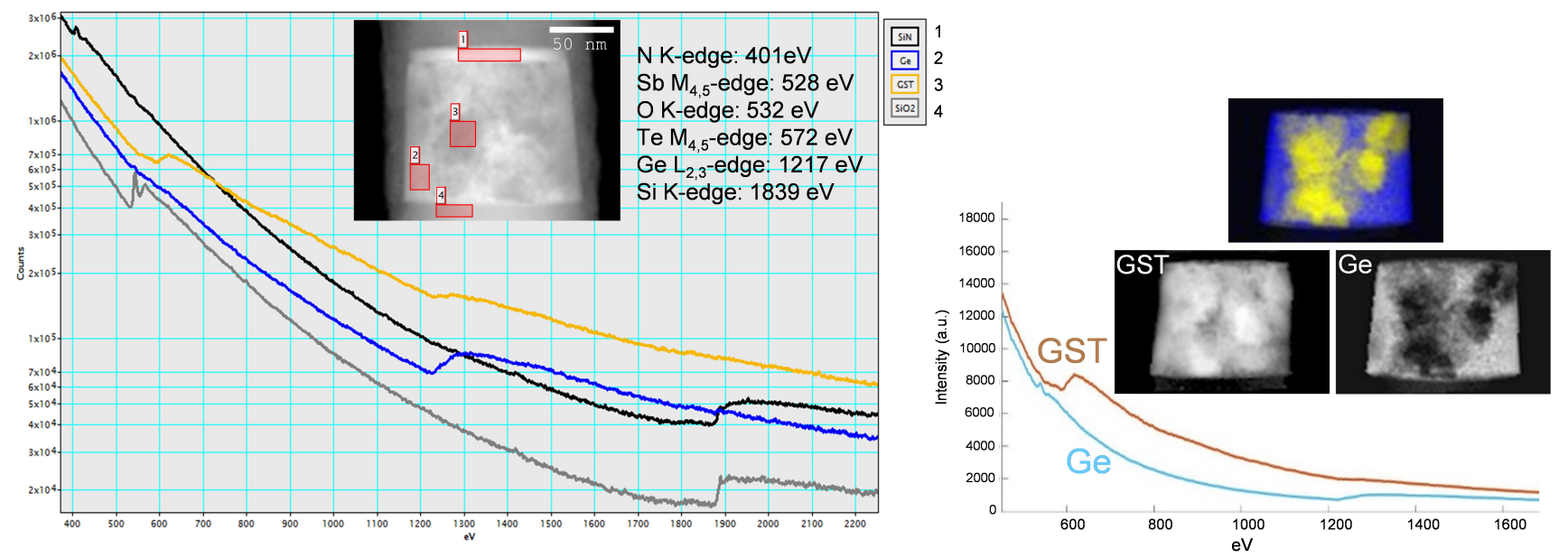

Figure 1. Left: HAADF-STEM image of the needle-shaped phase-change material (at $0^{\circ}$ ), and the EELS spectra of the regions marked in red in the inset, corresponding to $\mathrm{SiN}, \mathrm{SiO}_{2}, \mathrm{Ge}$ and GST. Right: VCA results showing the spectral components of the phases of interest (Ge and GST) and the corresponding maps (colored map: GST in yellow, Ge in blue).
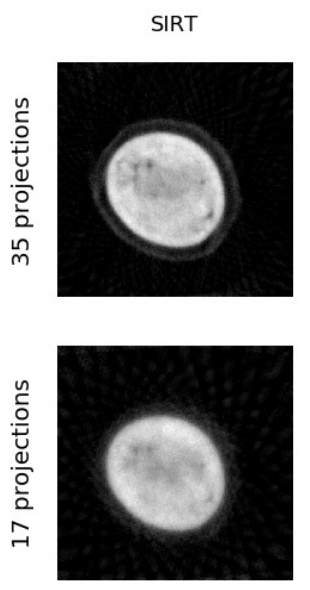
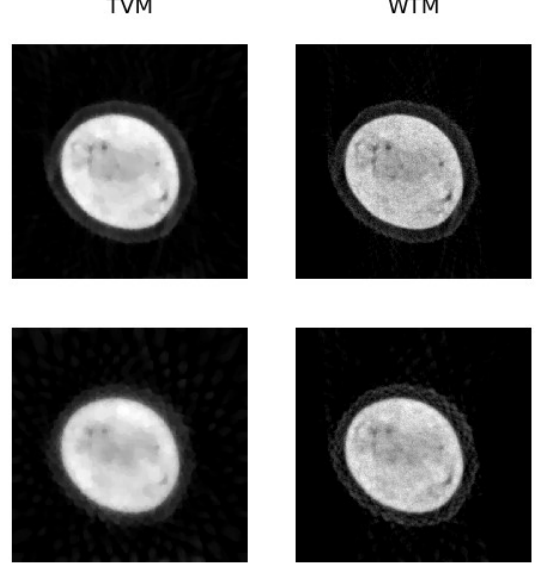

Figure 2. HAADF-STEM reconstruction. SIRT, TVM and wavelet-based CS reconstruction of a selected slice with 35 (top) and 17 (bottom) projections.

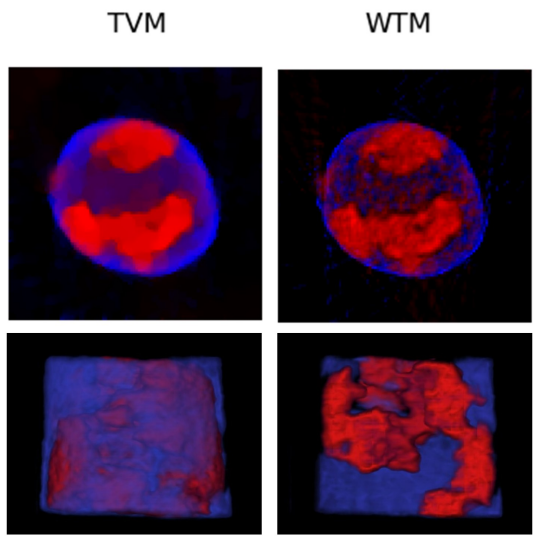

Figure 3. STEM-EELS reconstruction (GST in red, Ge in blue). Top: TVM and wavelet-based CS reconstruction of a selected slice. Bottom: volume rendering (left) and a slice through (right) the GST and Ge reconstructions with the waveletbased CS algorithm. 